\title{
Peripheral corneal ulcers associated with use of African traditional eye medicines
}

\author{
Susan Lewallen, Paul Courtright
}

\begin{abstract}
The most common cause of monocular blindness in Africa is corneal opacification. Traditional eye medicines (TEM) are widely used in Africa and their use has been associated with corneal ulceration, however, no controlled studies of the effects of TEM on the cornea have been published. We conducted a case-control study of 39 patients with corneal ulcers matched to controls with severe conjunctivitis. Microbiological investigations were conducted on 20 cases. There was a significant association between corneal ulceration and TEM use and, in particular, peripheral corneal ulcerations were significantly associated with TEM use.
\end{abstract}

(Brf Ophthalmol 1995; 79: 343-346)

Corneal disease is a major cause of blindness in developing countries; it is the most common cause of monocular blindness. In Africa, treatment of corneal disease is often delayed for weeks and patients frequently present to medical facilities with advanced disease including perforated ulcers and completely melted corneas. Use of traditional eye medicines (TEM) is common in Africa ${ }^{1-5}$ but little is known about these substances. In one survey in Malawi, $26 \%$ of blindness in blind schoolchildren was associated with use of TEM. ${ }^{2}$ In addition to damage caused directly by instillation of TEM into eyes, the use of these medicines leads to delay in presentation to hospitals and clinics. We have found that approximately $33 \%$ of patients with corneal disease who present to district hospitals (which refer patients to the tertiary hospital) have already used TEM; these patients take, on average, four times as long to present as those who do not use TEM. ${ }^{5}$

At the Queen Elizabeth Central Hospital (QECH) in Blantyre, Malawi, which serves as a tertiary referral hospital for 5 million people in the southern region, a number of patients with corneal disease are treated; among these are a large group which are similar and distinctive. These patients have peripheral ulcers, usually perforated, with filtering descemetoceles in otherwise quiet eyes. Although patients are often reluctant to admit to TEM use, it is frequently possible, by careful questioning, to elicit a history of symptoms which describe conjunctivitis, for which the patient used TEM.

To find out more about the association of TEM with corneal disease, and specifically with peripheral ulceration, we conducted a case-control study of patients with corneal ulcers presenting to QECH.

\section{Materials and methods}

Data were collected on patients admitted consecutively to the eye ward at QECH from January to September in 1992.

Cases were patients with an acute corneal ulcer who reported that the problem had begun within the previous month and who had no history of trauma. We excluded cases with trauma (which comprise approximately half of the admissions) because the traumatic episode itself would determine the location of the ulcer on the cornea. We also excluded any patient with a history of chronic or recurrent disease in the affected eye and ulcers we believed on clinical grounds to be due to herpes simplex.

Controls were patients admitted to the ward with severe conjunctivitis who had no staining of the cornea with fluorescein instillation. They were matched by age (within 5 years) to cases. Patients with conjunctivitis were chosen as controls because we wanted to compare corneal ulcer patients with a group with an external disease which would be expected to have signs and symptoms similar to corneal ulcers and to have a similar risk of exposure to the risk factor (use of TEM).

All cases and controls were interviewed by trained nurses using a standard pre-tested form to obtain information on initial symptoms, treatment sought (clinic or traditional healer), and response to this, and date of last use of TEM. TEM use was counted as positive for this episode if it had been used in the previous month.

All cases were examined by one ophthalmologist at a slit-lamp and findings were recorded on a standard form, noting the size, depth, and the location of the ulcer, whether the anterior chamber was formed, presence of hypopyon, and degree of inflammation and chemosis of the conjunctiva (graded as mild, moderate, or severe). Patients were examined every 2 to 3 days and progress of the ulcer recorded. Ulcers were classified as peripheral (those with an edge within $1 \mathrm{~mm}$ of the

Table 1 Cases and controls according to traditional eye medicine use

\begin{tabular}{lll}
\hline & \multicolumn{2}{l}{ Traditional eye medicines } \\
\cline { 2 - 3 } & Used & Not used \\
& No (\%) & No (\%) \\
\hline Corneal ulcers (cases) & $23(59 \cdot 0)$ & $16(41 \cdot 0)$ \\
Severe conjunctivitis (controls) & $3(7 \cdot 9)$ & $35(92 \cdot 1)$ \\
\hline
\end{tabular}

Differences are statistically significant at $\mathrm{p}<0.001$ (odds ratio $16 \cdot 8(95 \%$ confidence interval $3 \cdot 9,82 \cdot 7))$. 
Figure 1 Patient 7 (A) on presentation after use of traditional eye medicine, (B) 1 month later, (C) 2 months after presentation when the ulcer has healed with formation of a flat iridocorneal adhesion. (The inferotemporal corneal scar visible in all photographs is the site of previous trauma unrelated to the current problem.)
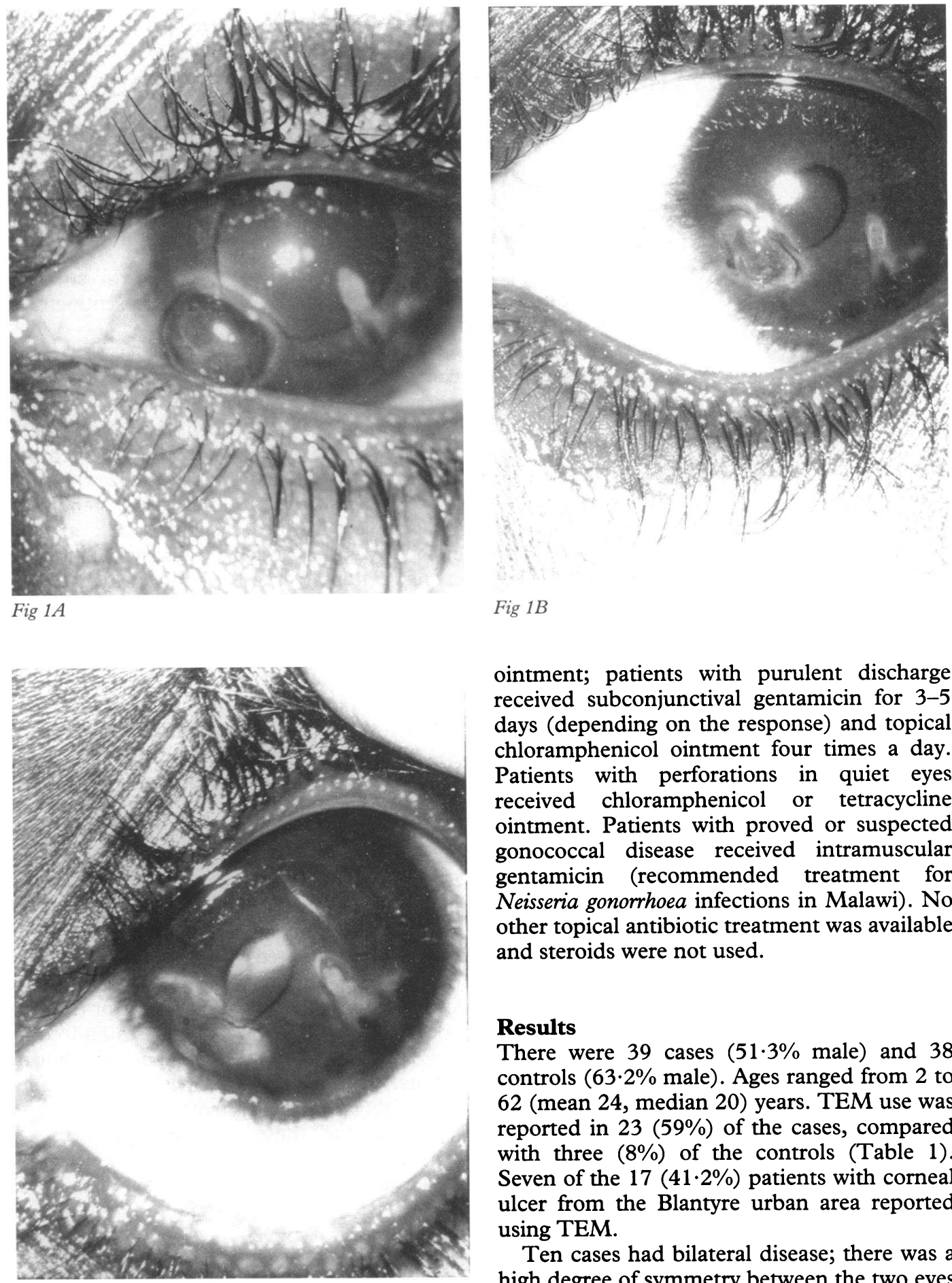

Fig $1 C$

limbus), central (those inside this limit), or entire cornea (those in which there was no cornea at all). We planned to culture the ulcers and the conjunctivae of all cases; however, laboratory support proved inadequate for this and it was completed in only the first 20 cases enrolled. The ulcer was scraped with a sterile platinum spatula and plated directly on blood agar and chocolate; cooked meat medium was also inoculated. In many of the perforated ulcers (or when the entire cornea was gone) it was not possible to scrape the cornea, so the conjunctiva was cultured by rolling a swab moistened with sterile broth in the fornices and plating this directly onto blood agar and chocolate.

Treatment in all cases included atropine ointment; patients with purulent discharge received subconjunctival gentamicin for 3-5 days (depending on the response) and topical chloramphenicol ointment four times a day. Patients with perforations in quiet eyes received chloramphenicol or tetracycline ointment. Patients with proved or suspected gonococcal disease received intramuscular gentamicin (recommended treatment for Neisseria gonorrhoea infections in Malawi). No other topical antibiotic treatment was available

\section{Results}

There were 39 cases $(51 \cdot 3 \%$ male $)$ and 38 controls (63.2\% male). Ages ranged from 2 to 62 (mean 24, median 20) years. TEM use was reported in $23(59 \%)$ of the cases, compared with three $(8 \%)$ of the controls (Table 1). Seven of the $17(41 \cdot 2 \%)$ patients with corneal ulcer from the Blantyre urban area reported using TEM.

Ten cases had bilateral disease; there was a high degree of symmetry between the two eyes in the appearance of the ulcers in these cases. Twenty three cases $(60.5 \%)$ were classified as peripheral ulcers (in five patients these were

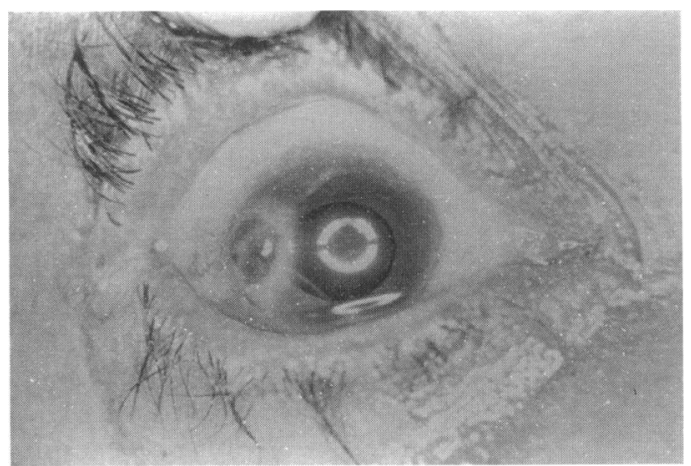

Figure 2 Filtering descemetocele in otherwise quiet eye of a patient who used traditional eye medicines. and steroids were not used. 
Table 2 Location of corneal ulcer according to use of traditional eye medicines

\begin{tabular}{|c|c|c|c|}
\hline & \multicolumn{3}{|c|}{ Traditional eye medicines } \\
\hline & $\begin{array}{l}\text { Used } \\
\text { No (\%) }\end{array}$ & $\begin{array}{l}\text { Not used } \\
\text { No (\%) }\end{array}$ & $\begin{array}{l}\text { Odds ratio }{ }^{\star}(95 \% \\
\text { confidence interval) }\end{array}$ \\
\hline $\begin{array}{l}\text { Peripheral cornea } \\
\text { Entire cornea } \\
\text { Central cornea }\end{array}$ & $\begin{array}{l}16(69 \cdot 6) \\
7(70 \cdot 0) \\
0(0)\end{array}$ & $\begin{array}{l}7(30 \cdot 4) \\
3(30 \cdot 0) \\
6(100)\end{array}$ & $\begin{array}{c}14.9(1.4,381 \cdot 9), p=0.004 \\
14.0(0.9,435.2), p=0.01 \\
1.0\end{array}$ \\
\hline
\end{tabular}

* Odds ratio calculated ( 1 has been added to each cell for calculation) with central cornea as the reference group $(O R=1 \cdot 0)$. Fisher's exact test (two tailed) $p$ value.

bilateral); 16 of these had perforated corneas in otherwise quiet eyes, such as are shown in Figures 1 and 2. The peripheral ulcer category included some patients in which most of the cornea was involved. However, the presence of a small central island with radial remnants of cornea was evidence that these had been peripheral ulcers initially. Furthermore, in two of these cases the fellow eye had a large peripheral perforation with a filtering descemetocele. Six patients had central ulcers and the remaining 10 patients had ulceration of the entire cornea (bilateral in five of these patients).

TEM use was reported in $16(69 \%)$ of the patients with peripheral ulcers, in none of those with central ulcers, and in seven $(70 \%)$ of those in which the entire cornea was ulcerated (Table 2). The results of the microbiological investigations are shown in Table 3.

\section{Discussion}

TEM use is common in Africa, in urban as well as rural areas. Because patients with corneal disease in Africa frequently present to tertiary care facilities late in the course of disease with inexplicit histories, it is difficult to sort out the relative contributions of trauma, TEM, bacterial, viral, or fungal infection to the disease. None the less, we have designed this study in an attempt to tease out the contribution of TEM, by enrolling only those patients with relatively acute disease and no history of trauma.

We found a significant association between
TEM use and peripheral ulcers. Our microbiological results are too varied to make associations between specific organisms and corneal ulcer appearance. The lack of fungal ulcers in this series is probably due to the fact that we excluded patients with a history of trauma, which usually accompanies fungal ulcers. It is interesting, however, that in several of the patients with peripheral perforations in relatively quiet eyes in which TEM had been used, the cultures were mixed and the organisms were relatively non-virulent. We believe that TEM was probably the primary agent responsible for these ulcers. In every case, these ulcers healed by formation of an iridocorneal adhesion, while the patient was observed in hospital.

The occurrence of adult keratoconjunctivitis due to $N$ gonorrhoea in this population has been documented previously. ${ }^{6}$ The cases in which $N$ gonorrhoea was cultured were always accompanied by extreme purulence, chemosis, and conjunctival injection, whether or not TEM had been used. The propensity of this organism to produce corneal ulceration is well known; it has been our experience that such ulcers are frequently peripheral. If we thus exclude the cases of gonococcal infection (five cases proved microbiologically and two which we suspected because of gross purulence and associated urethritis) a significant association between TEM use and peripheral ulceration remains.

The role of TEM in the patients in whom the entire cornea was ulcerated is less clear. Among the three cases who did not use TEM, cultures revealed $N$ gonorrhoea in two and Pseudomonas in the other. It might be that number of applications, dosage, or length of time over which TEM is used determine whether ulcers will be peripheral or increase in number and enlarge to involve the entire cornea; however, it was not possible to elicit such detailed histories from these patients. All of these eyes required evisceration.

It is interesting that investigators from Tanzania found corneal ulcers associated with TEM use to be more likely to cause dense

Table 3 Microbiological results and clinical characteristics of ulcers

\begin{tabular}{|c|c|c|c|c|c|c|}
\hline & Organisms & Location & Perforation & Conjunctiva & TEM used & Laterality \\
\hline \multirow[t]{2}{*}{$\begin{array}{l}1 \\
2\end{array}$} & \multirow{2}{*}{$\begin{array}{l}S \text { aureus, Klebsiella ozaenae } \\
S \text { aureus, diphtheroids, non-pathogenic Neisseria, } \\
\beta \text { haemolytic streptococci }\end{array}$} & Peripheral & No & ++ & Yes & $\mathrm{U}$ \\
\hline & & Peripheral & Yes & + & Yes & $\mathrm{U}$ \\
\hline 3 & CNS, Moraxella & Peripheral & Yes & ++ & Yes & $\mathrm{U}$ \\
\hline 4 & $S$ aureus, diphtheroids, Enterobacter agglomerans & Entire & Yes & +++ & Yes & $\mathrm{U}$ \\
\hline 5 & $S$ aureus, diphtheroids, non-pathogenic Neisseria & Peripheral & Yes & + & Yes & $\mathrm{U}$ \\
\hline 6 & Serratia marcescens, CNS & Peripheral & Yes & + & Yes & $\mathrm{U}$ \\
\hline 7 & CNS, diphtheroids & Peripheral & Yes & + & Yes & $\mathrm{U}$ \\
\hline 8 & $S$ aureus & Central & No & +++ & No & $\mathrm{U}$ \\
\hline 9 & Spneumoniae & Central & No & ++ & No & $\mathrm{U}$ \\
\hline 10 & $S$ pneumoniae, diphtheroids & Peripheral & Yes & + & Yes & $\mathbf{B}$ \\
\hline 11 & N gonorrhoea, CNS & Peripheral & Yes & +++ & Yes & $\mathrm{U}$ \\
\hline 12 & $S$ aureus & Peripheral & No & +++ & Yes & $\mathrm{U}$ \\
\hline 13 & $N$ gonorrhoea, CNS & Entire & Yes & +++ & No & $\mathbf{B}$ \\
\hline 14 & Pseudomonas & Entire & Yes & +++ & No & B \\
\hline 15 & N gonorrhoea & Entire & Yes & +++ & No & $\mathbf{B}$ \\
\hline 16 & N gonorrhoea & Peripheral & Yes & +++ & Yes & B \\
\hline 17 & N gonorrhoea & Entire & Yes & +++ & Yes & $\mathrm{U}$ \\
\hline 18 & CNS & Entire & Yes & +++ & Yes & $\mathrm{U}$ \\
\hline 19 & Pseudomonas & Entire & Yes & +++ & Yes & $\mathrm{U}$ \\
\hline 20 & CNS, $S$ aureus, Enterobacter aerogenes & Central & No & + & Yes & $\mathrm{U}$ \\
\hline
\end{tabular}

CNS = coagulase negative staphylococci. Entire=entire cornea.

Conjunctiva: degree of inflammation/chemosis, $+=$ mild, $++=$ moderate, $+++=$ severe

Laterality: $\mathrm{U}=$ Unilateral, $B=$ Bilateral. 
central scarring than ulcers with no associated TEM use. ${ }^{4}$ It is difficult to compare their findings with ours because their patients included both trauma cases and those with herpes simplex, which is much less common in our population. ${ }^{7}$ Nevertheless, it is clear that TEM is commonly used in their population and that it is associated with corneal ulceration.

TEM may cause corneal damage by introducing bacteria into the eye which lead to primary or secondary infections, or by toxic effects. We have interviewed over 100 traditional healers in southern Malawi (unpublished data) and have found that most 'drugs' used by these healers to treat eye disease consist of dried plant material, crushed into powder and made into aqueous solutions, or of infusions made from plants boiled in water. Animal products (including urine) are not used in this area. We hypothesise that an important component of the damage caused by TEM use is a toxic or perhaps immunologically mediated reaction which leads to a peripheral, often perforated, ulcer. This may heal by formation of an iridocorneal adhesion, as long as the TEM use is discontinued and secondary infection does not occur. If TEM treatment is continued or secondary infection occurs, however, the entire cornea may become ulcerated, resulting in a blind eye.

This work was supported by Project ORBIS and the International Eye Foundation.

1 Foster A, Sommer A. Corneal ulceration, measles, and childhood blindness in Tanzania. Br f Ophthalmol 1987; 71: $331-43$.

2 Chirambo M, Ben-Ezra D. Causes of blindness among students in blind school institutions in a developing country. Br f Ophthalmol 1976; 60: 665-8.

3 McMoli TE, Bordoh AN, Munube GMR, Bell EJ. Epidemic acute haemorrhagic conjunctivitis in Lagos, Nigeria. $\mathrm{Br} \mathcal{F}$ Ophthalmol 1984; 68: 401-4.

4 Yorston D, Foster A. Traditional eye medicines and corneal ulceration in Tanzania. F Trop Med Hyg 1994; 97: 211-4.

5 Courtright P, Lewallen S, Kanjaloti S, Divala DJ. Traditional eye medicine use among patients with corneal disease in rural Malawi. Br f Ophthalmol 1994; 78: 810-2.

6 Schwab L, Tizazu T. Destructive epidemic Neisseria gonorrhoea keratoconjunctivitis in African adults. $B r \mathcal{F}$ Ophthalmol 1985; 69: 525-8.

7 Lewallen S, Chirambo M. Herpetic corneal ulcers in Malawi. Br f Ophthalmol 1993; 77: 827. 